\title{
NONPARAMETRIC INDEXES FOR SENSITIVITY AND BIAS:
}

\section{COMPUTING FORMULAS}

\author{
J. BROWN GRIER ${ }^{1}$ \\ Northern Illinois University
}

\begin{abstract}
Computing formulas are derived for two nonparametric indexes of sensitivity and bias that have been suggested for signal detectability studies. A relationship is shown between the sensitivity index and $P(I)$, a statistic whose sampling variability is known. An additional index of bias is proposed, which is free of certain inconveniences, yet yields identical isobias contours. Use of the new indexes is illustrated with several sets of data.
\end{abstract}

Development of the theory of signal detectability has lead to a renewed interest in the possible processes involved in perception, psychophysics, and recognition memory. To a considerable extent both theory and research in these areas have rested on specific assumptions about the underlying distributions (as in the various threshold theories versus normality). But even without explicit assumption about the distributions, data are often judged by how close they lie to operating characteristic curves derived from normal distributions, and different experimental conditions are characterized by their value of $d^{\prime}$. Recently there has been a growing interest in various "nonparametric" analyses of detection/ recognition experiments, where specific underlying distributions are not assumed.

Following one line of development, Green (1964) has shown that for experiments using the yes-no procedure, the area under the (theoretical) operating characteristic curve can be interpreted as the percentage correct on an equivalent unbiased forced-choice test, and that this is true for any continuous underlying distributions. The sampling variability of this area measure has been determined by Pollack and Hsieh (1969). A similar proof for ratingscale experiments is due to Green and Moses (1966). However, using data to estimate the area under a curve of unknown theoretical shape presents difficulties. One expedient has been to connect the points and use the trapezoidal rule (Green \& Moses, 1966; Pollack, Norman, \& Galanter, 1964). If the true func-

\footnotetext{
${ }^{1}$ Requests for reprints should be sent to J. Brown Grier, Department of Psychology, Northern Illinois University, Dekalb, Illinois 60115.
}

tion is convex and the points precisely determined, this method is biased and will underestimate the true area; Simpson's rule should be better. However, exact area estimation will depend on having a functional relation between data and the area. Also, the area analysis, while sufficient to describe the data, does not preserve the notions of sensitivity and bias.

In a second approach to nonparametric analysis Pollack and Norman (1964) and Hodos (1970) have proposed measures based on the geometry of the unit square which can be interpreted as indexes of sensitivity and bias, respectively.

However, neither paper gives functional expressions for computing their index from the data, although Hodos does suggest a graphical estimation procedure. The purpose of the present paper is to derive explicit computing expressions for the indexes, their associated isosensitivity and isobias contours; to show the relationship of the new sensitivity index to the area measure; and to give examples of their use.

\section{COMPUTING EXPRESSIONS}

In the absence of specific assumptions about the underlying distributions, and hence the operating characteristic curves which could relate data points to the area, Pollack and Norman (1964) suggested computing an area statistic $A^{\prime}$ which is the average of the maximum and minimum possible areas associated with a point. Consider the outcome $P=(x, y)$ of a typical detection experiment plotted in Figure 1, where $x$ is the probability of a false alarm, and $y$ the probability of a hit. The two 


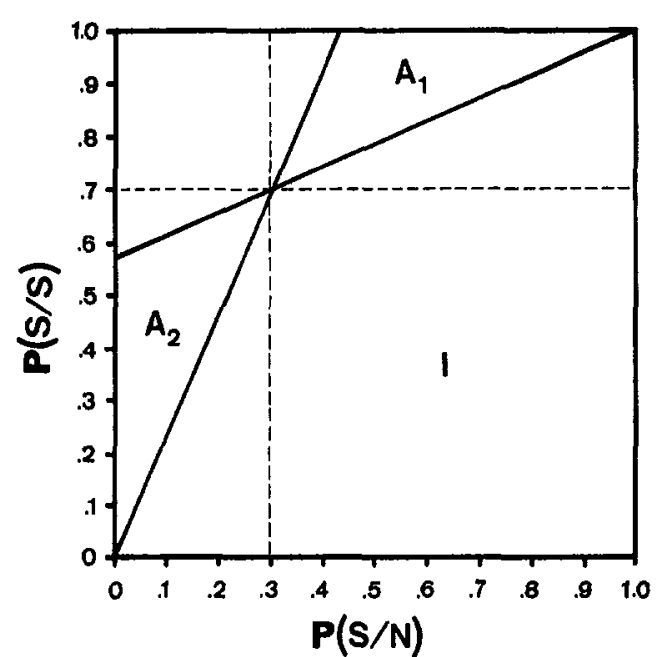

FIG. 1. Typical experimental outcome represented as a point in the unit square. The probability of a hit $[P(S / S)]$ is plotted against the probability of false alarm $[P(S / N)]$.

solid lines through the point and $(0,0),(1,1)$, respectively, form two nonoverlapping triangles, $A_{1}$ and $A_{2}$, which define the locus of all possible operating characteristic curves through the point. The four line segments then define upper and lower bounds for Green's area measure. Pollack and Norman's index is then

$$
A^{\prime}=I+\frac{1}{2}\left(A_{1}+A_{2}\right)
$$

where $I$ is the area under the solid lines. The index represents the average area under the upper and lower bounds.

Dividing the unit square as indicated by the solid and broken lines in Figure 1, and using the coordinates of the point $(x, y)$, the different areas in Equation 1 may be determined to give

$$
A^{\prime}=\frac{1}{2}+\frac{(y-x)(1+y-x)}{4 y(1-x)} \text {. }
$$

Solving for $y$ gives the expression for the isoperformance or isosensitivity curve

$\hat{y}=\min \left(1, \sqrt{\hat{x}(1-\hat{x})+(k / 2)^{2}}-(k / 2)\right)$

where

$$
k=3-4\left[\hat{x}+A^{\prime}(1-\hat{x})\right] .
$$

(Uncapped letters refer to data points, and capped letters to general values.) This curve can be interpreted as the locus of all points giving equivalent "average" performance.
Pollack and Norman (1964, Figure 2) show operating characteristic curves for several values of $A^{\prime}$. The curves are similar to normal operating characteristic curves for small values of $A^{\prime}$, but slightly flatter for large values.

The point of intersection with the negative diagonal can be determined as

$$
(\hat{x}, \hat{y})=\left[\frac{2\left(1-A^{\prime}\right)}{3-2 A^{\prime}}, 1-\frac{2\left(1-A^{\prime}\right)}{3-2 A^{\prime}}\right] .
$$

The value of $\hat{y}$ is $P(I)$ as described by Pollack and Hsieh (1969) who relate it to $d^{\prime}{ }_{e}$ a nonparametric sensitivity index suggested by Egan. The expression can be simplified to

$$
P(I)=\frac{1}{3-2 A^{\prime}}
$$

Other experimental outcomes along the isoperformance curve presumably differ from $P$ because of different criteria or bias.

Hodos (1970) has suggested as a nonparametric measure of bias the degree to which an outcome lies away from the negative diagonal. Referring again to Figure 1, now let $A_{1}$ and $A_{2}$ refer to the two triangles sharing a common right angle in the upper left corner. If his bias index is called $B^{\prime}{ }_{n}$, Hodos has proposed

$$
B^{\prime}{ }_{H}=\frac{A_{1}-A_{2}}{A_{1}}
$$

for points to the left of the negative diagonal, and the denominator changed to $A_{2}$ for points to the right. Again using the areas suggested in Figure 1, Hodos' index can be shown to be

$$
B^{\prime}{ }_{I I}=1-\frac{x(1-x)}{y(1-y)}
$$

for points to the left of the diagonal and

$$
B_{H}^{\prime}=\frac{y(1-y)}{x(1-x)}-1
$$

for points to the right. Solving Equation 7 gives the equation for the isobias contours:

$$
\hat{y}=\frac{1}{2} \pm \sqrt{\frac{1}{4}-\frac{\hat{x}(1-\hat{x})}{1-B^{\prime}{ }_{I I}}} .
$$

A family of these curves for different values of $B_{H}{ }^{\prime}$ is given in Figure 2 of Hodos (1970). 
TABLE 1

Analysis of Two Sets of Data from Green and Swets

\begin{tabular}{c|c|c|c|c|c|c}
\hline \hline$P(S / N)$ & $P(S / S)$ & $P(I)$ & $P(I)-.660$ & $\frac{P(I)-.660}{S D P(I)}$ & $P(I)-\overline{P(I)}$ & $\frac{P(I)-\overline{P(I)}}{S D P(I)}$ \\
\hline \multicolumn{1}{c|}{} & .335 & .066 & .006 & .04 & .003 & .02 \\
.205 & .510 & .663 & .003 & .02 & - & - \\
.400 & .715 & .661 & .001 & .01 & -.002 & .01 \\
.490 & .785 & .657 & -.003 & -.02 & -.007 & .05 \\
.690 & .925 & .669 & .009 & .06 & .006 & .04 \\
.040 & .245 & .640 & -.020 & -1.30 & .007 & .45 \\
.130 & .300 & .618 & -.042 & -2.73 & -.016 & -1.04 \\
.335 & .095 & .080 & .020 & 1.30 & .046 & 3.00 \\
.535 & .780 & .033 & -.027 & -1.75 & -.001 & -.06 \\
.935 & .975 & .598 & -.062 & -4.03 & -.036 & -2.33 \\
\hline
\end{tabular}

The change of formula for points to the left and right of the negative diagonal might be inconvenient, and another index is

$$
B^{\prime \prime}=\frac{A_{1}-A_{2}}{A_{1}+A_{2}}
$$

or the difference in the two areas divided by their sum. The computing expression for this is

$$
B^{\prime \prime}=\frac{y(1-y)-x(1-x)}{y(1-y)+x(1-x)} .
$$

This index ranges from +1 to -1 , but in a slightly different fashion. The solution for the contours is

$$
\hat{y}=\frac{1}{2} \pm \sqrt{\frac{1}{4}-\frac{\hat{x}(1-\hat{x})\left(1+B^{\prime \prime}\right)}{1-B^{\prime \prime}}}
$$

The two indexes put identical isobias contours through a given point, and choice between them seems to be a matter of convenience.

If two points along an isoperformance curve are thought of as differing due to a change of criteria, a more natural index of change might be the likelihood ratio criterion. The value of this index for each point can be determined from the derivative of Equation 3 as

$$
\begin{aligned}
B^{\prime} & =2\left(1-A^{\prime}\right) \\
& -\frac{1}{2} \frac{5-6 x-14 A^{\prime}+16 A^{\prime} x+8 A^{\prime 2}(1-x)}{\sqrt{x(1-x)+(k / 2)^{2}}}
\end{aligned}
$$

within the limits of the square.

\section{EXAMPLES}

Use of the formulas is illustrated by several examples. Two sets of data reported by Green and Swets (1966, p. 90) are reproduced in Table 1. The top half is from an auditory detection experiment in which the a priori probability of a signal was manipulated, and the lower half from the same experimental setting, but with the values of the decision outcomes varied. The data are plotted in Figures 2 and 3 along with the experimentally expected normal operating characteristic curve of $d^{\prime}=.85$. Visual inspection suggests isosensitivity for the data in Figure 2 and possible rejection for

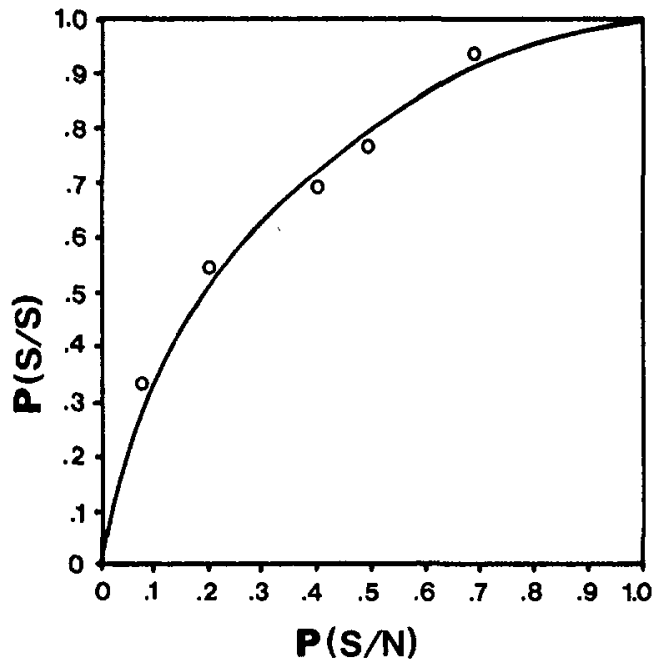

FIG. 2. Plot of data from a study by Green and Swets (see Table 1). 


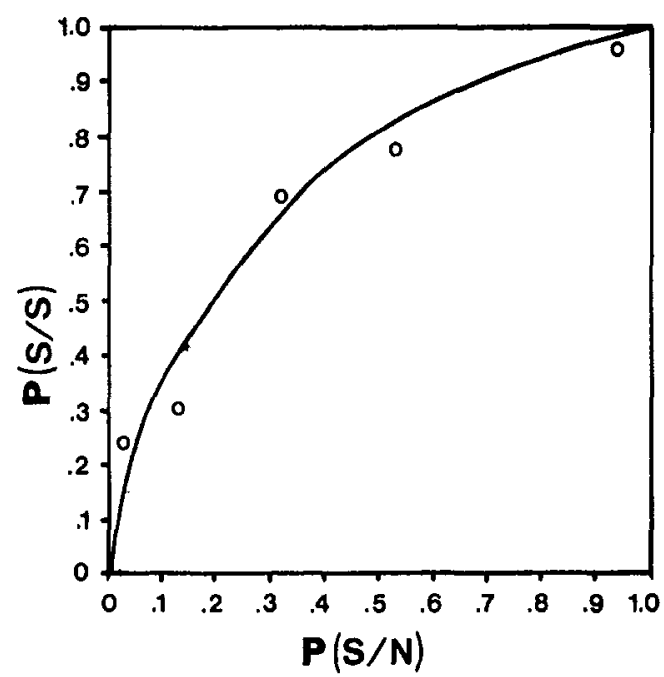

Fic. 3. Plot of data from a study by Green and Swets (see Table 1).

Figure 3. The nonparametric index is used to examine isosensitivity. In this case the nonparametric isosensitivity curve is virtually identical in shape to the normal, and the plotted curve can be taken as representing both. For each point the value of $P(I)$ was computed from Equation 5, and is given in column 3. The predicted value of the $P(I)$ based on a $d^{\prime}$ of .85 is approximately .660 . The deviations from the expected value are given in column 4. The standard deviation of $P(I)$ can be read from Figure 17 of Pollack and Hsieh (1969) as about .06 and correcting to $N=600$ on which the data are based gives $S D P(I)$ $=.0154$. The number of standard deviations of each point from the expected is given in column 5. For the first experiment the hypothesis of isosensitivity cannot be rejected. The second experiment requires further analysis. The lower half of column 5 shows that their data do not agree closely with the theoretically expected results. A second question is whether the data points represent a common sensitivity, even though different from the expected one. In the absence of more explicit techniques for combining multiple observations to estimate a common curve the individual $P(I) \mathrm{s}$ are averaged and $\overrightarrow{P(I)}=.634$. The deviation and the number of standard deviations from this value are given in columns 6 and 7, respectively. The fit to the average nonparametric
TABLE 2

Analysis of Data from Murdock

\begin{tabular}{c|c|c|c|c}
\hline \hline$P(S / N)$ & $P(S / S)$ & $P(I)$ & $d(x, y-N)$ & $d(x, y-N P)$ \\
\hline .053 & .460 & .7418 & .030 & .020 \\
.112 & .556 & .7407 & - & .006 \\
.212 & .673 & .7331 & -.020 & -.002 \\
.254 & .727 & .7364 & -.020 & - \\
.275 & .755 & .7496 & -.020 & - \\
.309 & .793 & .7440 & -.010 & .010 \\
.363 & .839 & .7452 & -.013 & .007 \\
.497 & .913 & .7353 & .005 & - \\
.576 & .957 & .7396 & .020 & - \\
\hline
\end{tabular}

curve is still poor. The center observation is three standard deviations from the mean and just about as far from its nearest neighbor. The hypothesis of a common isosensitivity curve of the Pollack and Norman type does not seem tenable.

Next, some data from a recognition memory experiment (Murdock, 1965, p. 445), which has been characterized by a normal operating characteristic curve, are examined. The data are reproduced in Table 2 and plotted in Figure 4 with the normal operating characteristic curve of $d^{\prime}=1.36$ estimated by Murdock as a solid line. The values of $P(I)$ for each point are in column 3 and their average is .7406 . No point appears to be an outlier, and the average nonparametric curve is plotted in Figure 4

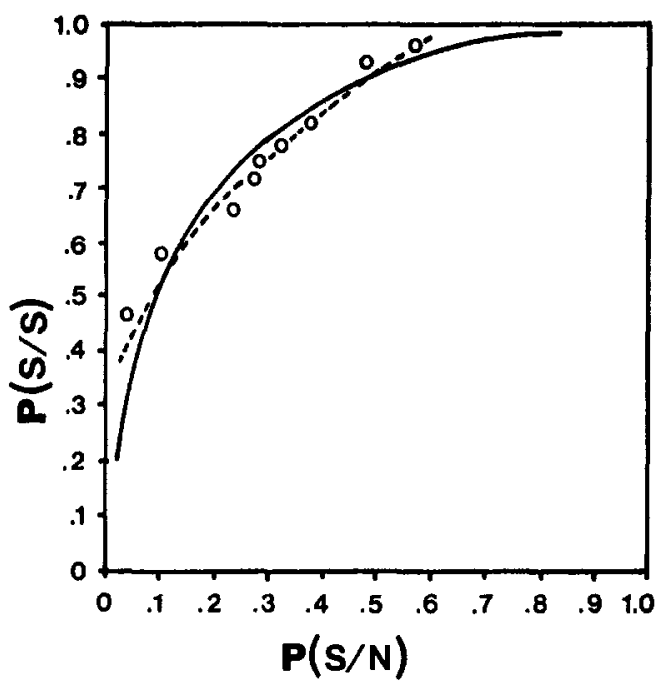

FIG. 4. Plot of data from a study by Murdock (see Table 2). 


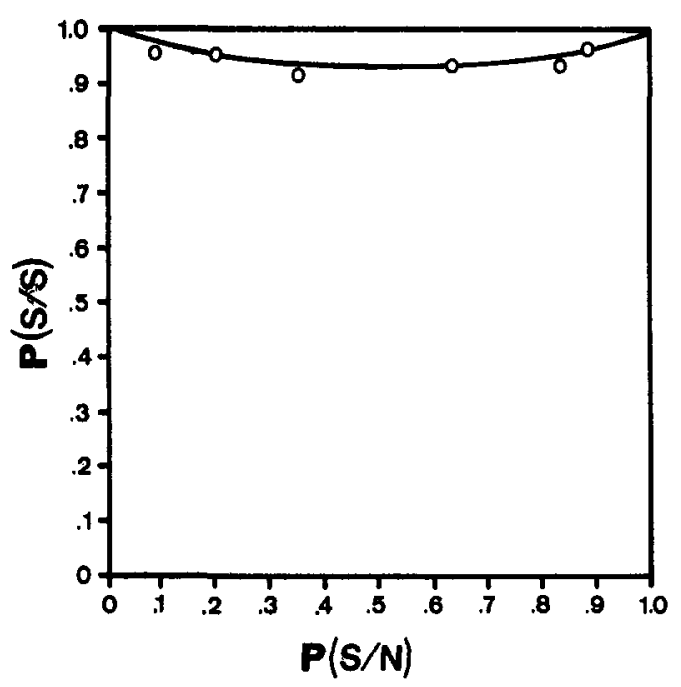

FIG. 5. Isobias contour from Hodos (see Table 3).

as the dashed line. Observation suggests the data lie closer to the nonparametric curve than to the normal. To examine fit, the data were plotted on an expanded graph, the two curves sketched, and the perpendicular distance from each point to the respective curves was measured with a pair of dividers, in units of the unit square. The signed deviations are given in Table 2.

The average deviation from the nonparametric operating characteristic curve is about one-third that from the normal (.0050 versus .0153 ).

A $2 \sigma$ interval around the mean of the unsigned nonparametric deviations includes zero $(.0050 \pm .0051)$, while the interval for the normal curve deviations does not (.0153 $\pm .0066)$. The nonparametric curve seems to provide a more satisfactory characterization of the data.

Use of the bias index is illustrated with some measurements from Figure 3A of Hodos (1970), part of which is reproduced in Figure 5. The data points lie close to the isobias contour. The location of each point was measured with dividers and is given in Table 3 along with values for $B^{\prime}{ }_{H}$ and $B^{\prime \prime}$. The significance of the observed differences is difficult to judge without sampling distributions, but the hypothesis of isobias suggested by the graph can be questioned. There may be some error of measurement since the original data were not available, but the example serves to illustrate
TABLE 3

Measured Locations in Hodos' Figure

\begin{tabular}{c|c|c|c}
\hline \hline$x$ & $y$ & $B^{\prime}{ }_{\boldsymbol{H}}$ & $B^{\prime \prime}$ \\
\hline .09 & .97 & -.645 & -.476 \\
.20 & .96 & -.760 & -.613 \\
.35 & .94 & -.752 & -.603 \\
.63 & .94 & -.758 & -.610 \\
.83 & .95 & -.663 & -.496 \\
.88 & .97 & -.724 & -.568 \\
\hline
\end{tabular}

the difficulty of making isobias judgments visually. The different isobias contours lie quite close together near the corners of the square.

\section{Discussion}

These derivations seem to provide new indexes that are both informative and easily computable for each data point. They are nonparametric only in the sense that no specific assumptions are made about the shape of the underlying distributions. For the sensitivity index a very specific assumption is made about the relationship between the distributions, but the assumed curve seems to fit some existing data at least as well and possibly better than symmetric normality. Since the assumed curve is symmetric, it might not be useful in experiments where the shape of the operating characteristic curve is important, or where results indicate assymmetry. The new statistics will be more useful when their sampling distributions are tabulated for use in making confidence statements, but, meanwhile, questions of homogeneity within groups, and differences between groups can be examined with available statistical procedures.

An analytical expression for the area under the nonparametric operating characteristic curve would be desirable because of its interpretation in terms of equivalent forced-choice performance. For a single point, one can be obtained by using Equation 3, but the integration must be done for two parts depending on whether $A^{\prime}$ is equal to or greater than .75 , and in either case gives extremely messy algebraic expressions which have not yielded to simplification. When multiple data points are available, the problem of combining them into a common area estimate will require statistical analysis. Simply computing an area 
for each point and then averaging the different areas may or may not prove an effective procedure.

\section{REFERENCES}

GREEN, D. M. General prediction relating yes-no and forced choice results. Journal of the Acousical Society of America, 1964, 36, 1042.

GreEN, D. M., \& Moses, F. L. On the equivalence of two recognition measures of short-term memory. Psychological Bulletin, 1966, 66, 228-234.

Green, D. M., \& Swets, J. A. Signal detection theory and psychophysics. New York: Wiley, 1966.
Hodos, W. A nonparametric index of response bias for use in detection and recognition experiments. Psychological Bulletin, 1970, 74, 351-354.

MURDOCK, B. B. Signal detection theory and short term memory. Journal of Experimental Psychology, $1965,70,443-447$.

PoLLACK, I., \& HSIEH, R. Sampling variability of the area under the ROC-curve and of $d^{\prime}{ }^{\circ}$. Psychological Bulletin, 1969, 71, 161-173.

Pol.LACK, I., \& NORMAN, D. A. A nonparametric analysis of recognition experiments. Psychonomic Science, 1964, 1, 125-126.

Pollack, I., Norman, D. A., \& Galanert, E. An efficient nonparametric analysis of recognition memory. Psychonomic Science, 1964, 1, 327-328.

(Received January 6, 1970)

\section{Manuscripts Accepted for Publication in the \\ Psychological Bulletin}

Explanation for Rewards that Do Not Reduce Tissue Needs: Robert Eisenberger*: Department of Psychology, University of California, Riverside, California 92502.

Evaluation of Research on Expectancy Theory Predictions of Employee Performance: Herbert G. Heneman III and Donald P. Schwab*: Graduate School of Business, 1155 Observatory Drive, University of Wisconsin, Madison, Wisconsin 53706.

Use of Rank Order Data in Functional Measurement: David J. Weiss and Norman H. Anderson*: Department of Psychology, University of California at San Diego, La Jolla, California 92037.

Influence of Economic and Political Ideologies upon the Development of Developmental Psychology: Klaus F. Riegel*: Department of Psychology, University of Michigan, Ann Arbor, Michigan 48104.

Rapid Eye Movements and Visual Imagery during Sleep: David Koulack*: Department of Psychology, University of Manitoba, Winnipeg, Canada.

Response to Cronbach and Furby's "How Should We Measure Change-Or Should We?": Edward F. O'Conner, Jr.*: Educational Testing Service, Princeton, New Jersey 08540.

Some Comments on "A Behavioral Approach to Nitrogen Narcosis": Barry Fowler": Institute for Environmental Medicine, University of Pennsylvania, Philadelphia, Pennsylvania 19104.

Test of Normality Against Skewed Alternatives: Ralph B. D'Agostino* and Edward E. Cureton: Department of Mathematics, Boston University, Charles River Campus, Boston, Massachusetts 02215.

\footnotetext{
* Asterisk indicates author for whom the address is supplied.
} 